\title{
COVID-19 mimicking ST-elevation myocardial infarction
}

\author{
Kamil Bujak, Anna Kazik, Michał Wróbel, Jacek Piegza, Andrzej Lekston, Mariusz Gąsior \\ $3^{\text {rd }}$ Department of Cardiology, Faculty of Medical Sciences in Zabrze, Medical University of Silesia in Katowice, Poland
}

Adv Interv Cardiol 2020; 16, 2 (60): 213-215

DOI: https://doi.org/10.5114/aic.2020.95632

A 71-year-old woman with a history of previous left anterior descending artery (LAD) angioplasty, non-ST-elevation myocardial infarction 13 years earlier (treated medically), and hypertension, presented with persistent, midsternal, non-radiating chest pain for several hours, and was admitted to our center during the outbreak of coronavirus disease 2019 (COVID-19) in Poland. She was transported to our hospital by emergency medical service after electrocardiogram (ECG) e-transmission, which demonstrated ST-segment elevation in inferior leads, and ST depression, and inverted T waves in V1-3 (Figure $1 \mathrm{~A}$ ). During the transport to the hospital, she was started on a loading dose of ticagrelor and unfractionated heparin. On arrival, she was afebrile; blood pressure was 140/ $95 \mathrm{~mm} \mathrm{Hg}$, and heart rate was $88 / \mathrm{min}$. She denied dyspnea and cough. However, the patient and other members of her family had influenza-like symptoms for 2 weeks prior to admission. She was brought to the catheterization laboratory immediately, where coronary angiography was performed and revealed non-obstructive coronary artery disease and no in-stent restenosis in the LAD (Figures 1 $B, C)$. After the procedure, the patient was transferred directly to the department dedicated to patients with suspected or confirmed COVID-19 at our center. The initial and subsequent high-sensitivity troponin I levels were negative. C-reactive protein and D-dimer levels were mildly elevated, whereas other blood test results, including complete blood count, were within the normal range. Transthoracic echocardiogram demonstrated preserved left ventricular ejection fraction of $50 \%$ with inferior and septal hypokinesis. A chest X-ray showed no pulmonary opacities (Figure $1 \mathrm{D}$ ). Due to previous influenza-like symptoms, a nasopharyngeal swab (PCR testing) was performed and was positive for severe acute respiratory syndrome coronavirus 2 (SARS-CoV-2). Considering that the patient's status was good with only mild symptoms, she was discharged from the hospital and transferred to the isolation unit on the third day of admission.

The current COVID-19 pandemic is an unprecedented and challenging situation for all medical specialties. While most patients with SARS-CoV-2 infection present with respiratory symptoms, cardiovascular manifestations of COVID-19 are also observed in a substantial proportion of patients and are usually associated with poor prognosis [1, 2]. In the cohort of patients hospitalized for COVID-19 in Wuhan, China, approximately twenty percent of all patients had a cardiac injury, but only 3.4\% complained of chest pain [1]. Most frequently, myocardial injury in patients with COVID-19 seems to be associated with myocarditis or cytokine storm [1, 2]. Increased risk of developing type 1 myocardial infarction (systemic inflammation predisposes to plaque rupture) and type 2 (related to oxygen supply and demand imbalance) is also possible [1, 3]. Takotsubo syndrome and spontaneous coronary artery dissection have also been observed in patients with SARS-CoV-2 infection $[3,4]$. None of these conditions were found; moreover, the diagnostic criteria for acute pericarditis were not met in this case. Therefore we could not state with certainty that there was a causal relationship between either ischemic ECG changes or an episode of chest pain and COVID-19 in this patient. During the COVID-19 pandemic, the management of patients suspected of acute myocardial infarction needs to be careful and responsible, in order to reduce SARS-CoV-2 spread.

\section{Conflict of interest}

The authors declare no conflict of interest. 
A

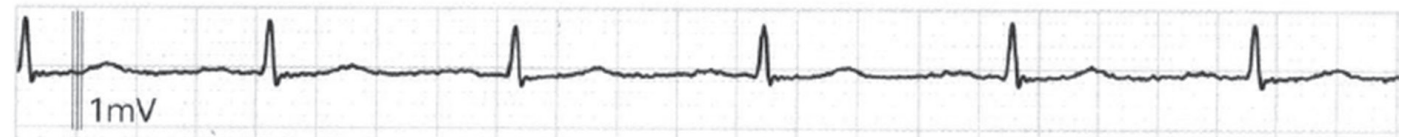

II

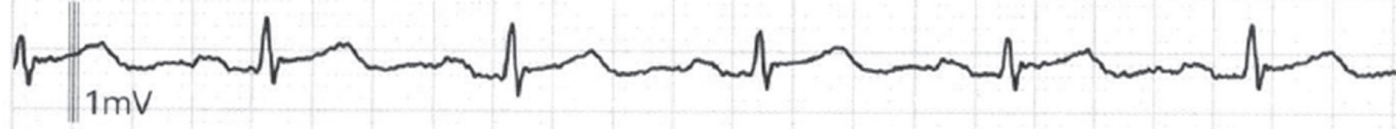

*III

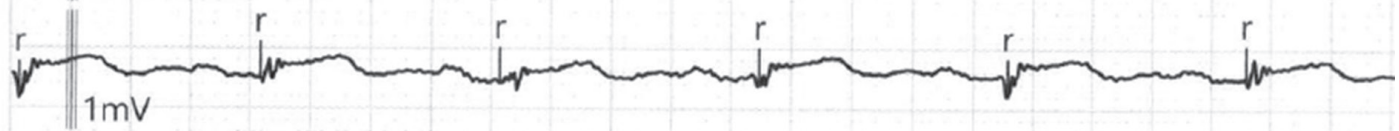

aVR

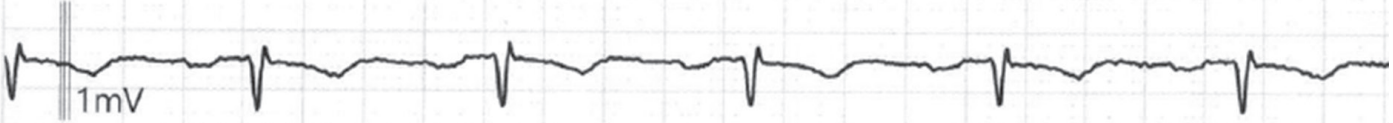

$\mathrm{aVL}$

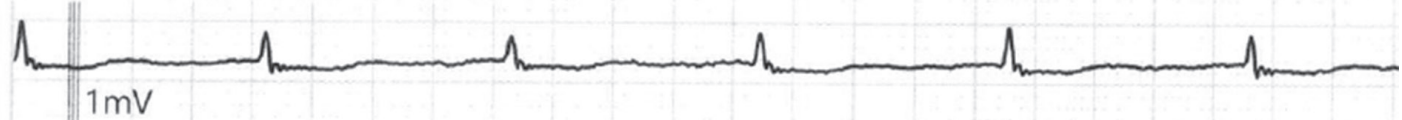

aVF

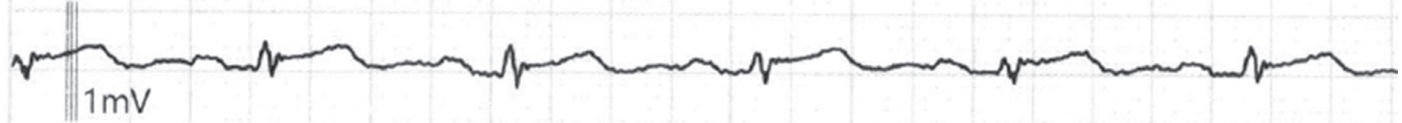

V1
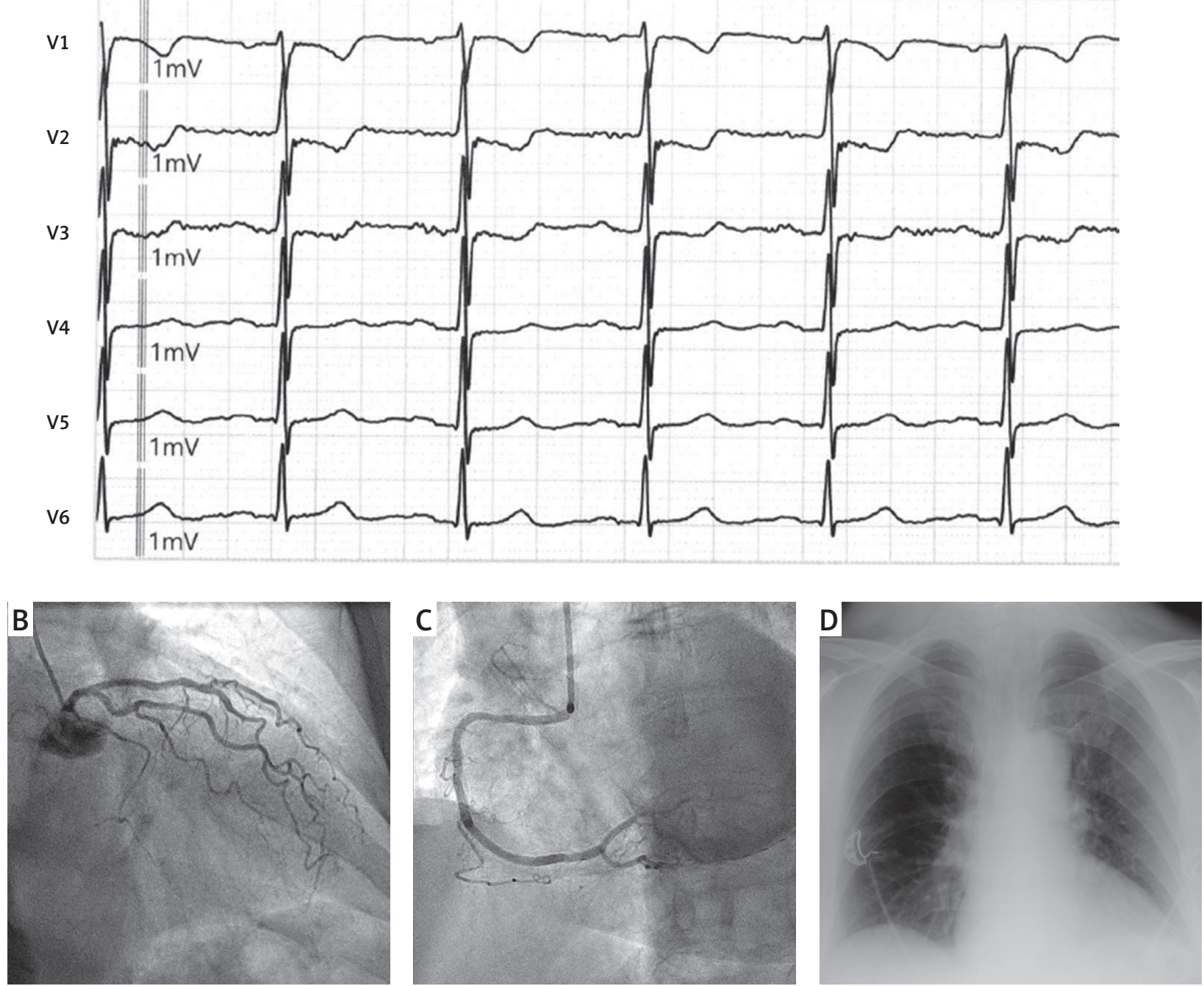

Figure 1. A - 12-lead electrocardiogram demonstrating ST-segment elevation in inferior leads, and ST depression and inverted T waves in V1-3. B - Left coronary angiogram showing non-obstructive stenoses in circumflex and left descending artery and no in-stent restenosis. C - Right coronary angiogram showing non-significant lesions. D - Chest X-ray revealing no pulmonary opacities 


\section{References}

1. Shi S, Qin M, Shen B, et al. Association of cardiac injury with mortality in hospitalized patients with COVID-19 in Wuhan, China. JAMA Cardiol 2020; DOI: 10.1001/jamacardio.2020.0950.

2. Fried JA, Ramasubbu K, Bhatt R, et al. The variety of cardiovascular presentations of COVID-19. Circulation 2020; 141: 1930-6.

3. Courand PY, Harbaoui B, Bonnet M, Lantelme P. Spontaneous coronary artery dissection in a patient with COVID-19. JACC Cardiovasc Interv 2020; DOI: 10.1016/j.jcin.2020.04.006.

4. Meyer P, Degrauwe S, Van Delden C, et al. Typical takotsubo syndrome triggered by SARS-CoV-2 infection. Eur Heart J 2020; 41: 1860. 